\title{
HIV and T follicular helper cells: a dangerous relationship
}

\author{
Carola G. Vinuesa
}

Department of Pathogens and Immunity, John Curtin School of Medical Research, Australian National University, Canberra, Australia.

\begin{abstract}
HIV infection leads to progressive destruction of infected CD4 T cells, hypergammaglobulinemia, and loss of memory B cells. Germinal centers, which are key to memory $B$ cell formation and protective antibody responses, are major HIV reservoirs in which the virus replicates within $\mathrm{T}$ follicular helper (TFH) cells. In this issue of the JCI, the Koup and Streeck groups report that chronic SIV/HIV infection promotes TFH cell accumulation, which may drive B cell dysregulation. Their discoveries suggest that HIV harnesses TFH cells to evade the antibody response.
\end{abstract}

Antibodies play an essential role in the protection against viral infection, acting through different effector mechanisms to prevent viral entry into host cells and eliminate infected cells. A few viruses, however, have evolved sophisticated mechanisms to evade the neutralizing antibody response and therefore persist. In the case of HIV-1, multiple mechanisms enable it to evade antibody binding and replicate at leisure. These mechanisms include the death of virus-specific CD4 T cells that are required to help the $\mathrm{B}$ cell response, a high mutation rate that allows the rapid emergence of antigenic escape variants, and a structure of the envelope complex that makes conserved epitopes relatively inaccessible to antibodies $(1,2)$. In addition to preventing otherwise effective antibodies from inhibiting virus entry into cells, HIV can also alter the quality of the antibody response: chronic HIV infection is associated with hypergammaglobulinemia, poor antibody responses to protein vaccines, and decreased antigen-specific memory B cells (reviewed in ref. 3 ). The studies in this issue of the JCI by Lindqvist et al. (4) and Petrovas et al. (5), together with a parallel study by Hong et al. (6), suggest that accumulation of $\mathrm{T}$ follicular helper (TFH) cells, together with possibly altered function during chronic HIV infection, may be at the root of this form of humoral evasion.

\section{Germinal center origin of protective anti-HIV antibodies}

$B$ cells that receive cognate $T$ cell help can differentiate into antibody-producing

Conflict of interest: The author has declared that no conflict of interest exists.

Citation for this article: J Clin Invest. 2012; 122(9):3059-3062. doi:10.1172/JCI65175. cells along two separate routes. An extrafollicular pathway generates short-lived, low-affinity plasmablasts, whereas the follicular route gives rise to specialized microenvironments called germinal centers, in which affinity maturation of the antibody response takes place (7). Within germinal centers, antigen-specific B cells acquire random point mutations targeted at the $\mathrm{Ig} \mathrm{V}$ region genes that change the specificity of the $\mathrm{B}$ cell receptor. Those $\mathrm{B}$ cells that consequently bind antigen with higher affinity are thought to gain competitive advantage to receive selection signals from specialized TFH cells. Selected B cells terminally differentiate into longlived memory B cells or memory plasma cells that home to the bone marrow (7).

Despite the described resistance of HIV to antibody-mediated neutralization, up to $25 \%$ of HIV-1-infected individuals manage to develop high titers of broadly neutralizing antibodies over time. The high-affinity human antibody VRC01, which recognizes the initial site of CD4 attachment on HIV-1 GP120 and neutralizes about $90 \%$ of HIV-1 isolates, appears to evolve from a low-affinity unmutated germline ancestor via the acquisition of a vast number -70 to 90 - of somatic mutations (8). This represents around a 4-fold higher frequency of changes than those typically observed during the affinity maturation process. These and other data have suggested a germinal center origin and TFH-mediated selection of broadly neutralizing antibodies and raise the possibility that altered selection in germinal centers may contribute to the failure to generate protective antibodies in infected individuals.

\section{HIV infects and expands TFH cells}

It has been known since the late 1980s that germinal centers constitute the largest reservoirs of HIV virions (9), which are retained in the form of immune complexes on the processes of follicular dendritic cells (FDCs) (10). It has also been suggested that active viral infection occurs primarily at these sites during clinical latency (11). Viral replication does not occur in FDCs themselves, but rather in germinal center CD4 T cells, now known as TFH cells (12-14). TFH susceptibility to SIV infection is also confirmed in the current study by Petrovas and colleagues (5). There have even been suggestions that HIV-1 may replicate more actively in germinal center TFH cells compared with that in other CD4 T cells (15).

In the context of murine LCMV infection, viral persistence promotes the accumulation of TFH cells in mouse secondary lymphoid tissues, which helps control infection (16). Now three groups also report TFH cell accumulation during SIV and HIV infection. Petrovas et al. observed that the proportion of TFH cells was increased by approximately 8 fold in $50 \%$ of chronically SIV-1-infected rhesus macaques (RMs) designated "TFH hi" - compared with that in uninfected or acutely infected RMs or the "TFH ${ }^{\mathrm{lo}}$ " group of chronically infected RMs (5). Hong and colleagues enumerated TFH cells per unit area of lymph node sections; an approximately 4-fold increase in TFH cells was also observed in chronically infected macaques compared with that in uninfected or acutely infected ones (6). These results suggest an increase in TFH cells in absolute numbers, as opposed to a lower rate of depletion compared with other CD4 T cell subsets. In the study by Lindqvist et al., HIV-infected individuals had approximately 10 -fold higher frequencies of TFH cells compared with those of uninfected subjects (4). This is in itself quite extraordinary since HIV kills the vast majority of cells it infects (17), and, as discussed above, TFH cells themselves are susceptible to infection. None of the groups observed a correlation between 


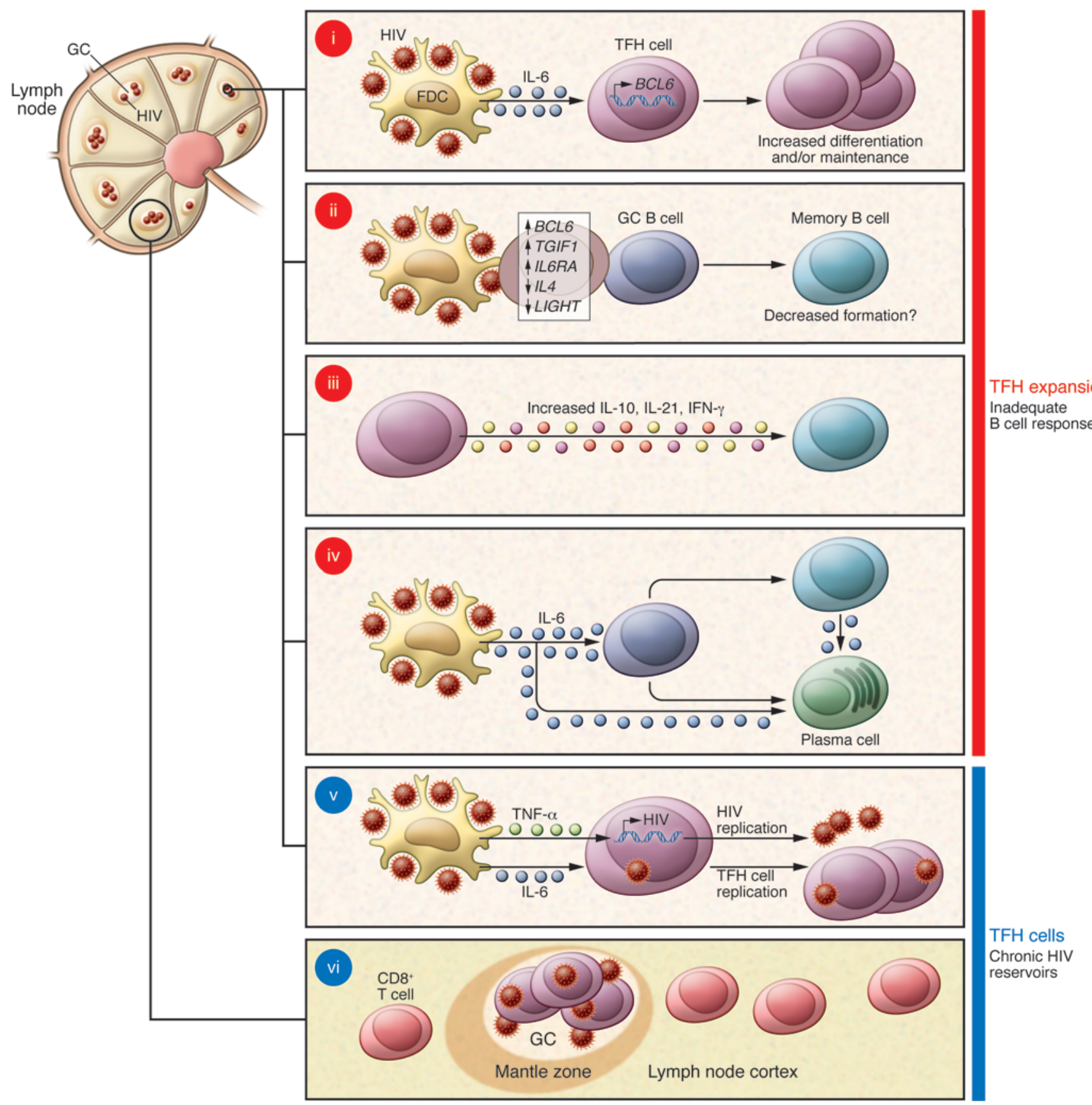

Figure 1

Potential mechanisms by which TFH cells drive B cell dysregulation and constitute privileged virus reservoirs in HIV infection. (i) Excessive IL-6 produced by FDCs that harbor HIV viruses leads to upregulation of BCL-6 and increased differentiation and/or maintenance of TFH cells. Increased TFH cell numbers, together with abundant antigen, lowers competition for B cell selection reducing affinity maturation. (ii) Chronic virus infection changes the TFH cell expression of key molecules, including BCL6, IL6RA, IL4, LIGHT, and TGIF1, leading to altered function that may contribute to decreased memory B cell formation. (iii) In HIV-infected individuals, a larger fraction of TFH cells secrete IL-21 and possibly other cytokines known to promote B cell differentiation and antibody production; this can contribute to the hypergammaglobulinemia. (iv) The high IL-6 levels found in HIV infection may directly promote plasmacytosis and even enhance differentiation of memory B cells into plasma cells, leading to hypergammaglobulinemia and loss of memory B cells. (v) HIV replicates in TFH cells, and this may be enhanced by FDC-derived cytokines, including TNF. Exposure to high levels of IL-6 might also explain the increased proliferative rate of TFH cells in infected individuals, which may help maintain the viral reservoirs. (vi) It has been suggested that infection of TFH cells may be a strategy for viral persistence, since germinal centers are relatively devoid of cytolytic CD8 ${ }^{+} \mathrm{T}$ cells. GC, germinal center. 
HIV-1 plasma viral load and TFH cell numbers, although it is possible that such correlation might exist with tissue virus levels; virus is known to persist in tissues even when it is undetectable in the blood (18).

What drives the TFH cell accumulation in chronic HIV infection? First, TFH cells extracted from HIV-infected individuals expressed more BCL- 6 - the transcription factor required for $\mathrm{TFH}$ formation and maintenance (19) - than those from treated or uninfected subjects (4) (Figure 1, i). In the context of chronic LCMV viral infection, TFH cell accumulation occurs as a consequence of IL- 6 produced by a radioresistant cell (16), and FDCs are a plausible candidate for this role within germinal centers. IL-6 mediates upregulation of BCL6 on antigen-specific CD4 T cells (19). IL-6 has been reported to be increased in HIV infection (20), and levels are elevated in the plasma from infected macaques (5). Consistent with a prominent role for IL- 6 in driving TFH cell expansion, IL-6 receptor signaling was increased in SIV-infected RMs with high frequency of TFH cells: IL-6R $\alpha$ was found expressed at increased levels on TFH cells (Figure 1, ii). Furthermore, TFH cells had increased phosphorylation of STAT3, which mediates IL-6 receptor signaling (16).

\section{Aberrant TFH expansion and function may contribute to $B$ cell abnormalities}

Accumulation of TFH cells has been shown to maintain increased numbers of germinal center B cells and cause the prototypic systemic autoimmune disease SLE (21), probably due to the lowered threshold of $B$ cell selection in germinal centers. Intriguingly, and as Moir and Fauci have pointed out, virtually the same B cell abnormalities have been found in HIV and SLE (3). The explanation for this similarity may again lie in the accumulation of TFH cells: the three studies discussed here noted that the increase in TFH cells in chronically infected subjects was accompanied by parallel increases in germinal center B cells. Also, a positive correlation was observed between total IgG and both the proportion of TFH cells and BCL- 6 expression on these cells (4). TFH cell accumulation during HIV infection, together with excess antigen held on FDCs, may lower the threshold for $B$ cell selection in germinal centers and lead to the emergence of low-affinity variants (Figure 1, i). Lindqvist et al. observed that a larger fraction of TFH cells secreted IL-21 in untreated individuals compared with that in treated individuals (4). It is therefore possible that excess of this and other TFH-derived cytokines, such as IFN- $\gamma$ and IL-10, contributes to hypergammaglobulinemia (Figure 1, iii). Furthermore, the increased amounts of IL- 6 found in chronic HIV infection are likely to increase plasma cell survival and thus augment total IgG levels (Figure 1, iv).

Another intriguing observation was the inverse correlation between the proportion of TFH cells and memory B cells (4). There are at least two different explanations for this effect. First, it is likely that TFH cell accumulation is accompanied by functional changes in TFH cells, and this may impair germinal center $B$ cell selection and terminal differentiation (Figure 1, ii). This is suggested by the fact that the TFH/memory B cell inverse correlation was only observed in HIV-infected individuals but not in uninfected individuals (4) and by the observed differences in gene expression between TFH cells from infected and uninfected macaques (5). For example, the observed upregulation of TGIF1, which limits TGF- $\beta$-responsive gene expression, may act in a manner analogous to the depletion of follicular Tregs, which has been shown to lead to expanded germinal centers containing increased numbers of non-antigen-specific GC B cells (22). Also, downregulation of LIGHT, which costimulates CD40 triggering (23), may dampen memory B cell formation; CD40 signals have been reported to bias the differentiation of germinal center B cells toward memory rather than plasma cells (24). Increased expression of IL-6Ra and BCL- 6 in TFH cells is also likely to confer functional changes. The other possibility is that excessive IL- 6 signals directly to memory B cells and promotes their terminal differentiation into plasma cells (Figure 1 , iv); precedents for this action of IL-6 have been previously described (25). Therefore, it is possible that memory B cell formation is intact, but further differentiation into plasma cells causes memory B cell depletion (Figure 1,iv).

\section{Perspectives}

Collectively, these studies suggest that overactivity and possibly dysregulation of TFH cells explains the crippling B cell defects associated with HIV infection. It's also tempting to speculate that this TFH cell abnormality underpins the overrepresentation of autoimmune conditions and autoantibody development in HIV infection (26). A key outstanding issue is whether the TFH cell expansion seen in chronic HIV infection is beneficial or detrimental in mounting a high-affinity, broadly neutralizing antibody response against HIV in humans. TFH cell expansion was shown to help control chronic LCMV viral infection in mice (16) and correlate with production of high-affinity anti-SIV IgG antibodies in macaques (5). However, finding that the TFH cell expansion in humans favored accumulation of GAG-specific rather than envelope GP120-specific T cells (4) - broadly neutralizing antibodies are typically directed against structures in envelope - suggests that this TFH cell response may not be terribly helpful in controlling the infection. The implications of the recent discoveries for understanding HIV latency are also potentially exciting. Germinal centers store large quantities of HIV virus held on FDCs. The finding that TFH cells, which are susceptible to HIV infection, accumulate in lymph nodes rather than being depleted, suggests these cells may constitute privileged latent reservoirs (Figure 1, v). It has been suggested that the paucity of SIV-specific CD8 ${ }^{+} \mathrm{T}$ cells in follicles may protect infected TFH cells from being killed (ref. 6 and Figure 1, vi). Considerable effort is currently being directed toward the development of treatments that activate gene transcription to eliminate HIV latent reservoirs. It is conceivable that these or other antiretroviral drugs could be targeted to germinal center TFH cells by coupling them to antibodies such as PD-1. Finally, monitoring memory TFH-like cells in the blood has proven a useful biomarker of TFH cells and germinal center dysregulation in secondary lymphoid tissues in the context of autoimmunity (27). Given the parallels with chronic HIV infection, it is likely that circulating TFH-like cells may be a useful biomarker of the size of the HIV chronic reservoirs and provide a rationale for using TFH-targeted therapies.

\section{Acknowledgments}

C.G. Vinuesa is supported by NHMRC project and program grant funding and an NHMRC Elizabeth Blackburn Fellowship.

Address correspondence to: Carola G. Vinuesa, Department of Pathogens and Immunity, John Curtin School of Medical Research, Australian National University, Building 131, Garran Road, Acton, Canberra, ACT 0200, Australia. Phone: 61.26. 1254500; Fax: 61.26.1254595; E-mail: Carola. Vinuesa@anu.edu.au. 
1. Johnson RP. Mechanisms of protection against simian immunodeficiency virus infection. Vaccine. 2002;20(15):1985-1987.

2. Kwong PD, et al. HIV-1 evades antibody-mediated neutralization through conformational masking of receptor-binding sites. Nature. 2002; 420(6916):678-682.

3. Moir S, Fauci AS. B cells in HIV infection and disease. Nat Rev Immunol. 2009;9(4):235-45.

4. Lindqvist M, et al. Expansion of HIV-specific T follicular helper cells in chronic HIV infection. J Clin Invest. 2012;122(9):3271-3280.

5. Petrovas C, et al. CD4 T follicular helper cell dynamics during SIV infection. J Clin Invest. 2012; 122(9):3281-3294.

6. Hong JJ, Amancha PK, Rogers K, Ansari AA, Villinger F. Spatial alterations between CD4(+) T follicular helper, B, and CD8(+) T cells during simian immunodeficiency virus infection: $\mathrm{T} / \mathrm{B}$ cell homeostasis, activation, and potential mechanism for viral escape. J Immunol. 2012;188(7):3247-3256.

7. Vinuesa CG, Tangye SG, Moser B, Mackay CR. Follicular B helper $\mathrm{T}$ cells in antibody responses and autoimmunity. Nat Rev Immunol. 2005;5(11):853-865.

8. Wu X, et al. Focused evolution of HIV-1 neutralizing antibodies revealed by structures and deep sequencing. Science. 2011;333(6049):1593-1602.

9. Ringler DJ, et al. Cellular localization of simian immunodeficiency virus in lymphoid tissues. I. Immunohistochemistry and electron microscopy.
Am J Pathol. 1989;134(2):373-383.

10. Keele BF, et al. Characterization of the follicular dendritic cell reservoir of human immunodeficiency virus type 1. J Virol. 2008;82(11):5548-5561.

11. Heath SL, Tew JG, Tew JG, Szakal AK, Burton GF. Follicular dendritic cells and human immunodeficiency virus infectivity. Nature. 1995;377(6551):740-744.

12. Tenner-Racz K, von Stemm AM, Gühlk B, Schmitz J, Racz P. Are follicular dendritic cells, macrophages and interdigitating cells of the lymphoid tissue productively infected by HIV? Res Virol. 1994; 145(3-4):177-182.

13. Haase AT. Population biology of HIV-1 infection: viral and CD4+ T cell demographics and dynamics in lymphatic tissues. Annu Rev Immunol. 1999;17:625-656.

14. Gratton S, Cheynier R, Dumaurier MJ, Oksenhendler E, Wain-Hobson S. Highly restricted spread of HIV-1 and multiply infected cells within splenic germinal centers. Proc Natl Acad Sci U S A. 2000; 97(26):14566-14571.

15. Thacker TC, et al. Follicular dendritic cells and human immunodeficiency virus type 1 transcription in CD4+ T cells. J Virol. 2009;83(1):150-158.

16. Harker JA, Lewis GM, Mack L, Zuniga EI. Late interleukin-6 escalates $\mathrm{T}$ follicular helper cell responses and controls a chronic viral infection. Science. 2011;334(6057):825-829.

17. Mattapallil JJ, Douek DC, Hill B, Nishimura Y, Martin $\mathrm{M}$, Roederer M. Massive infection and loss of memory CD4+ T cells in multiple tissues during acute SIV infection. Nature. 2005;434(7037):1093-1097.

18. Cohen J. HIV/AIDS research. Tissue says blood is misleading, confusing HIV cure efforts. Science. 2011;334(6063): 1614

19. Crotty S. Follicular helper CD4 T cells (TFH). Annu Rev Immunol. 2011;29:621-663.

20. Kuller LH, et al. Inflammatory and coagulation biomarkers and mortality in patients with HIV infection. PLoS Med. 2008;5(10):e203.

21. Linterman $M$, et al. Follicular helper $T$ cells are required for systemic autoimmunity. J Exp Med. 2009;206(3):561-576.

22. Linterman MA, et al. Foxp3(+) follicular regulatory $\mathrm{T}$ cells control the germinal center response. Nat Med. 2011;17(8):975-982.

23. Duhen T, Pasero C, Mallet F, Barbarat B, Olive D, Costello RT. LIGHT costimulates CD40 triggering and induces immunoglobulin secretion; a novel key partner in T cell-dependent B cell terminal differentiation. Eur J Immunol. 2004;34(12):3534-3541.

24. Arpin C, et al. Generation of memory B cells and plasma cells in vitro. Science. 1995;268(5211):720-722.

25. Beagley KW, et al. Peyer's patch B cells with memory cell characteristics undergo terminal differentiation within 24 hours in response to interleukin-6. Cytokine. 1991;3(2):107-116.

26. Zandman-Goddard G, Shoenfeld Y. HIV and autoimmunity. Autoimmun Rev. 2002;1(6):329-337.

27. Vinuesa CG, Cook MC. Blood relatives of follicular helper T cells. Immunity. 2011;34(1):10-12. 